\title{
Influence of physiologic hormonal modification and of hormonal treatment in a patient with a history of migraine with aura
}

\author{
Ferdinando Maggioni - Antonio Palmieri • \\ Michele Tropea · Giorgio Zanchin
}

Received: 12 December 2007/Revised: 18 January 2008/ Accepted: 18 January 2008/Published online: 12 February 2008

(C) Springer-Verlag 2008

\begin{abstract}
We present the case of 48-year-old woman suffering from migraine with aura (MA) since menarche. During her life the patient frequently presented catamenial MA attacks with an increasing frequency during pregnancy in particular in the second and third trimesters, and then during breast-feeding. Treatment with oral contraceptive (OC) for endometriosis and later with cyclophosphamide, methotrexate, fluorouracil (CMF) for breast cancer produced a higher number of attacks. Instead, she referred an improvement with gonadotropin releasing hormone agonist (GnRH-a) for the treatment of endometriosis and then with tamoxifen as hormonal therapy after mastectomy and chemotherapy for breast cancer. We highlight the importance of physiological hormonal modification and hormonal therapies on the course of MA.
\end{abstract}

Keywords Migraine with aura - Tamoxifen .

Gonadotropin releasing hormone agonist .

Oral contraceptive $\cdot$ Female sex hormones

\section{Introduction}

The influence of ovarian hormone activity on migraine with (MA) and without aura (MO) throughout the female lifecycle is well known [1, 2]. Estrogenic production induces dichotomy in the incidence of migraine between

F. Maggioni $(\bowtie) \cdot$ M. Tropea $\cdot$ G. Zanchin

Headache Centre, Department of Neurosciences,

University of Padua, Padua, Italy

e-mail: ferdinando.maggioni@unipd.it

A. Palmieri

Headache Centre, Hospital San Donà di Pia (ve), Venice, Italy the two genders at the time of puberty, which becomes wider during the childbearing age and diminishes after menopause [1]. In women, the attacks often begin at menarche and then occur maximally in correlation with menses as pure menstrual migraine or menstrual-related migraine in a large amount of patients [3, 4]. Indeed, a change in migraine pattern is frequently observed during pregnancy [5, 6], puerperium [7] and after menopause [8, 9], and is related to hormonal therapies [10]. We present the case of a woman with a 36-year history of migraine with aura and describe the effect on the attacks of the hormonal physiological modifications and of the various hormonal therapeutic treatments that rarely could be found in a single individual.

\section{Case report}

A 48-year old woman presented at our Headache Centre for a long-standing history of migraine with aura (ICHD-II) [11]. She experienced her first episode by the age of 12 years, a few months after menarche. The attacks were characterized by gradual onset of flickering lights localized over the temporal aspect of both visual fields with an average duration of 30-40 min, followed within a halfhour by bilateral, throbbing and moderate to severe headache, accompanied by nausea, photophobia and phonophobia with a duration of about $6 \mathrm{~h}$. At the beginning, the attacks usually occurred during the menstrual period with a frequency of one episode every other month. Other trigger factors were reported, including emotional stresses and difficulties with study and, later on, with her work as a clerk. The frequency of attacks remained quite unchanged until the age of 26 when she became pregnant. During the second and third trimester, the patient 
experienced an increased frequency with at least two episodes a month, or more, in the last month prior to delivery. The attacks remained unchanged during puerperium and the 4 months of breast-feeding, after which the episodes persisted with a frequency of once a month. At the age of 31 , the patient was diagnosed with ovarian endometriosis for which she was started on oral contraceptives (OC). During the 6-month period of OC therapy, the attacks markedly worsened, presenting with an almost weekly frequency. For this reason, a prophylactic therapy with lisuride at the dosage of $0.025 \mathrm{mg}$ tid was added, without relevant improvement. Afterwards, the OC therapy was stopped and the patient underwent a partial ovariectomy. In the subsequent 6 months, she was treated with the gonadotropin-releasing hormone agonist $(\mathrm{GnRH}-\mathrm{a})$ triptoreline at the dosage of $3.75 \mathrm{mg}$ im once monthly, during which period the patient did not present with any attack. Nevertheless, at the end of treatment at the age of 33, the MA attacks recurred with high frequency. At that time, the patient experienced important emotional distress due to a severe disease suffered by her mother. At the age of 40 , she was diagnosed with a breast cancer. A radical mastectomy was performed and pathology showed a ductal adenocarcinoma with metastatic invasion of the axillary lymphonodes; estrogen and progesterone receptors were positive. The surgery was followed by six cycles of chemotherapy with cyclophosphamide, methotrexate and 5fluorouracil. During chemotherapy, the patient reported about three crises monthly, of which one was regularly, at the end of every cycle. After the end of chemotherapy, tamoxifen $20 \mathrm{mg}$ daily was started and the therapy lasted for 5 years. In the 5 years of tamoxifen therapy, the patient experienced only three attacks, but soon after the stop of therapy MA built up again with two or three episodes a month of severe headache preceded by visual disturbance and paresthesia, the latter being very unusual until then. A brain MR with gadolinium and MR-angiography were unremarkable. Migraine prophylactic treatment was prescribed with flunarizine $5 \mathrm{mg}$ daily for 6 months and then amitriptyline $50 \mathrm{mg}$ at bedtime for 8 months with poor efficacy.

\section{Discussion}

This case considers the milestones of the physiological hormonal life of a woman and their effects on the course of MA. Moreover, the pathologies presented by the patient (endometriosis and breast cancer) allow to evidence the importance of the treatments performed, in particular those with influence on hormonal activity, on MA attacks. The data, regarding the frequency of the crises are precise because, for many years before presenting to our Headache Centre, the patient entered in her detailed personal diary the events of her life, including the occurrence of the attacks and of menses. Clearly, the trigger factors for MA are not only hormonal, but also emotional, causes (the disease of her mother, the neoplasia, problems in the job). However, the relationships with the physiological hormonal modifications are very evident: first of all, we notice the beginning of the attacks a few months after menarche and the occurrence of the attacks often in relation to menses. Then, she developed a worsening of the attacks at the beginning of the third month of pregnancy, an unusual but not extraordinary circumstance in MA, that respect to migraine without aura (MO) may show a less favourable course or that sometimes develops during pregnancy [12]. Finally, we noted a persistence of the attacks during puerperium and breast-feeding. Regarding pharmacological treatments, we evidenced drugs that caused worsening or improvement of the attacks. In the first instance, there was a remarkable worsening of the crisis with the use of $\mathrm{OC}$, in spite of the concomitant prophylactic treatment. Moreover, although it is difficult to establish the exact mechanism of action by which antiblastic drugs might provoke the attacks at the end of every treatment, a cause-effect relationship appears clear. In the second instance, the treatment with GnRH-a for therapy of endometriosis and then with tamoxifen for adjuvant treatment of the neoplasia proved to be very effective in the prevention of the attacks, particularly tamoxifen that was prescribed for 5 years. The patient reported not more than three attacks during the entire period of anti-oestrogenic treatment. About tamoxifen, there are two studies in the literature [13, 14]. In an open-label non-randomized study on eight women suffering from not further specified catamenial migraine, five patients reported marked improvement or disappearance of headache, two mildmoderate improvement and one was unchanged. Tamoxifen was given at a dosage of $10-20 \mathrm{mg} /$ days for $7-$ 14 days before menstruation and continued at $5-10 \mathrm{mg}$ days for 3 days after the start of menstruation for four cycles [13]. The second report was on a series of six women with "migraine-type headaches" and benign mammary dysplasia treated with tamoxifen $20 \mathrm{mg} /$ day. Improvement was seen in all patients who no longer required migraine drug therapy, but the attacks returned a few weeks after tamoxifen discontinuation of the drug [14]. In contrast with these data, one case report of catamenial migraine showed a worsening of the attacks with tamoxifen [15]. We found two studies on the use of GnRH-a with partially divergent conclusions [16, 17]. The first was a non-randomized prospective study, in which five patients with menstrual migraine were treated with $3.75 \mathrm{mg}$ IM GnRH-a monthly for 10 months. For the 
last 6 months of treatment, patients also received transdermal estrogen and medroxyprogesterone $2.5 \mathrm{mg}$. All the patients reported a marked reduction of the attacks with leuprolide and with leuprolide and hormonal add-back therapy [16]. Conversely, Martin et al. [17] in a randomized, placebo-controlled study on 21 patients, treated with GnRH-a and with GnRH-a associated with estrogens, demonstrated a modest preventive benefit only in the group treated with $\mathrm{GnRH}-\mathrm{a}$ (goserelin $3.6 \mathrm{mg}$ implant) associated with a transdermal patch containing $100 \mu \mathrm{g}$ of oestradiol-17 $\beta$. The above reported studies, the only ones present in literature on this topic to the best of our knowledge, consider the use of tamoxifen and GnRHa only in the treatment of MO. We highlight the possible use of these treatments also in MA, but this needs further studies. A possible caveat is that often the course of MA is irregular with long periods of spontaneous remission and this pattern could make testing the efficacy of a therapy difficult. But this statement is not applicable to our patient who presented a rather regular course of the crisis. The uncommon behavior of MA in this patient should be stressed. MA is commonly thought to worsen in periods of high plasma estrogen concentration, such as the last two trimesters of pregnancy, but not with estrogen withdrawal. In this case, MA worsened with both low and high estrogen levels (i.e., menstrual period, post-partum, pregnancy). It seems that both hormonal fluctuations and stable high estrogen levels could trigger MA attacks, whereas stable low estrogen levels, obtained pharmacologically by anti-estrogen and GnRH-a drugs, could be beneficial. In our case, these therapies were given, incidentally, to treat other diseases (endometriosis and breast cancer) as in the Powles' report for benign mammary dysplasia [8]. We believe, considering these results, that in particular and selected situations, when the crises are particularly related to hormonal changes and difficult to treat, or when associated with concomitant gynecologic diseases, these drugs could be considered for use, although not as a first-line treatment, but with caution and in cooperation with a gynecologist.

\section{References}

1. Silberstein SD (2001) Headache and female hormones: what you need to know. Curr Opin Neurol 14:323-323

2. Zacur HA (2006) Hormonal changes throughout life in women. Headache 46(S2):S49-S54

3. Granella F, Sances G, Allais G, Nappi RE, Tirelli A, Benedetto C, Brundu B, Facchinetti F, Nappi G (2004) Characteristics of menstrual and non menstrual attacks in women with menstrually related migraine referred to headache centres. Cephalalgia 24:707-714

4. Ashkenazi A, Silberstein S (2007) Menstrual migraine:a review of hormonal causes, prophylaxis and treatment. Expert Opin Pharmacother 8:1605-1613

5. Maggioni F, Alessi C, Maggino T, Zanchin G (1997) Headache during pregnancy. Cephalalgia 17:765-769

6. Granella F, Sances G, Pucci E, Nappi RE, Ghiotto N, Nappi G (2000) Migraine with aura and reproductive life events: a case control study. Cephalalgia 20:701-707

7. Marcus DA, Scharff L, Turk D (1999) Longitudinal prospective study of headache during pregnancy and postpartum. Headache 39:625-632

8. Fettes I (1999) Migraine in the menopause. Neurology 53(S1):S29-S33

9. Neri I, Granella F, Nappi R, Manzoni GC, Facchinetti F, Genazzani AR (1993) Characteristics of headache at menopause: a clinico-epidemiologic study. Maturitas 17:31-37

10. Mac Gregor EA (2004) Oestrogen and attacks of migraine with and without aura. Lancet Neurol 3:354-361

11. Classification Comitee of the International Headache Society. (2004) The international classification of headache disorders (2nd edn). Cephalalgia 24:1-160

12. Cupini ML, Matteis M, Torisi E, Calabresi P, Berrnardi G, Silvestrini M (1995) Sex hormone related events in migrainous female A clinical comparative study between migraine with aura and without aura. Cephalalgia 15:140-144

13. O'Dea JP, Davis EH (1990) Tamoxifen in the treatment of menstrual migraine. Neurology 40:1323

14. Powles TJ (1986) Prevention of migrainous headache by tamoxifen. Lancet 2:1344

15. Mathew P, Fung F (1999) Recapitulation of menstrual migraine with tamoxifen. Lancet 353:467-468

16. Murray SC, Muse KN (1997) Effective treatment of severe menstrual migraine headaches with gonadotropin-releasing hormone agonist and add back therapy. Fertil Steril 67:390-393

17. Martin V, Wernke S, Mandell K, Zoma W, Bean J, Pinney S, Liu J, Ramadan N, Rebar R (2003) Medical oophorectomy with, without estrogen add-back therapy in the prevention of migraine headache. Headache 43:309-321 